\title{
Effects of Radiation and Chemical Reaction on Transient Free Convective MHD Flow over a Porous Plate
}

\author{
P. Srikara Reddy \\ Lect. in Mathematics, \\ Krishna Chaithanya Degree College, Nellore, A.P. \\ Dr. J. Girish Kumar \\ Lect. in Mathematics, \\ Govt. Degree College, Jammalamadugu, Kadapa, A.P. \\ Dr. R.V.S. Prasad \\ Reader in Statistics, \\ S.V.G.S. Degree College, Nellore, A.P
}

\begin{abstract}
The present chapter study sought to investigate the effects of radiation and chemical reaction as well as viscous heat dissipation on the transient free convection and mass transfer flow of an electrically conducting, viscous, incompressible fluid, past an infinite vertical porous plate, in presence of uniform externally applied transverse magnetic field. The plate is subjected to a variable suction velocity and both the temperature as well as concentration is assumed to be oscillating with time. The dimensionless governing equations for this study are solved numerically using finite difference method. The velocity, temperature and concentration profiles are shown graphically for various material parameters such as magnetic parameter (M), Prndtl number (Pr), Schmidt number (Sc), Chemical reaction parameter (k), Grashof number (Gr), modified Grashof number (Gc), Eckert number (Ec), radiation parameter (R).
\end{abstract}

Keywords - MHD, viscous dissipation, vertical porous plate, radiation, chemical reaction etc.

\section{INTRODUCTION}

Significant attention of many researchers is take place on the study of unsteady MHD free convection flow with mass transfer past a vertical porous plate due to its various applications viz. studies of plasma, extraction of geothermal energy, metallurgy, chemical, mineral and petroleum engineering, etc. and on the performance of so many engineering devices using electrically conducting fluids, namely, MHD generators, MHD pumps, MHD accelerators, MHD flow-meters, nuclear reactors, plasma jet engines, etc. Free convective MHD flow past an accelerated vertical porous plate was investigated by Singh (1) in this he used the finite difference method. Ahmmed et al. (2) gave the analytical solution to MHD free convection and mass transfer flow past a vertical porous plate. Das et al. (3) investigated the influences of mass transfer in the presence of oscillatory suction on MHD flow past a vertical porous plate embedded in a porous medium and heat source.

The influences of radiative MHD free convective flow past a vertical porous plate plays a vital role. Ahmmed et al. (4) investigated the numerical studies on MHD free convective mass transfer flow past a vertical flat plate. Abdussattar and Hamid Kalim (5) considered the unsteady free convective and thermal radiative fluid flow past a vertical porous plate. Makinde (6) discussed the mass transfer and free-convective, thermal radiative fluid flow past a moving vertical porous plate. Abd EL-Nabyet al (7) examined the finite difference solution for the unsteady radiative MHD free-convective fluid flow on vertical porous plate. Mbeledoguet al. (8) studied the compressible unsteady MHD free convection fluid flow past a moving vertical plate taking into the account of radioactive heat transfer.

Muthucumaraswamy and Chandrakala (9) studied the radiative heat and mass transfer effects on isothermal vertical plate taking into the account of chemical reaction. Ibrahim et al (10) discussed the effect of the chemical reacting 
and radiative fluid flow on the unsteady MHD free convection past a semi-infinite vertical permeable moving plate along with heat source and suction. Venkateswarlu and Padma (11) investigated the solution for unsteady MHD free convective heat and mass transfer flow past a vertical permeable plate while the consideration of thermal radiation and chemical reaction.

The aim of the present chapter is to analyze the effects of radiation on transient MHD free convection flow of an electrically conducting, viscous incompressible fluid over a porous vertical plate with homogeneous chemical reaction of first order. The equations of momentum, energy and diffusion, which govern the flow field, are solved by explicit finite difference method. The behavior of the velocity, temperature and concentration has been discussed for variation in the governing parameters.

\section{MATHEMATICAL FORMULATION}

The radiative and transient MHD free convection flow of an electrically conducting, viscous incompressible fluid over a porous vertical infinite plate with homogeneous chemical reaction of first order has been considered. The $\bar{x}$ axis is assumed to be taken along the plate and the $\bar{y}$-axis normal to the plate. Since the plate is considered infinite in $\bar{x}$ - direction, hence all physical quantities will be independent of $\bar{x}$. Under these assumption, the physical variables are functions of $\bar{y}$ and $\bar{t}$ only. The wall is maintained at constant temperature $\bar{T}_{w}$ and concentration $\bar{C}_{w}$ higher than the ambient temperature $\bar{T}_{\infty}$ and concentration $\bar{C}_{\infty}$ respectively. The fluid considered here is gray, absorbing - emitting radiation but a non - scattering medium. The Joule heating effects are assumed to be negligible in the energy equation. Also, it is assumed that there exists a homogeneous chemical reaction of first order with rate constant $\bar{k}$ between the diffusing species and the fluid. A uniform magnetic field of magnitude $B_{0}$ is applied normal to the plate. The transverse magnetic field and magnetic Reynolds number are assumed to be very small, so that the induced magnetic field is negligible. Also there is no applied voltage, so that the electric field is absent. The concentration of the diffusing species in the binary mixture is assumed to be very small in comparison with the other chemical species, which are present, and hence the Sorret and Dufour effects are negligible. Under the Boussinesq approximation and boundary layer theory, the governing equations for the problem under consideration are:

Continuity equation

$$
\frac{\partial \bar{u}}{\partial \bar{y}}=0
$$

Momentum equation

$$
\frac{\partial \bar{u}}{\partial \bar{t}}+\bar{v} \frac{\partial \bar{u}}{\partial \bar{y}}=g \beta\left(\bar{T}-\overline{T_{\infty}}\right)+g \beta^{*}\left(\bar{C}-\overline{C_{\infty}}\right)+\bar{v} \frac{\partial^{2} \bar{u}}{\partial \bar{y}^{2}}-\frac{\sigma B_{0}^{2}}{\rho} \bar{u}
$$

Energy equation

$$
\frac{\partial \bar{T}}{\partial \bar{t}}+\bar{v} \frac{\partial \bar{T}}{\partial \bar{y}}=\frac{\kappa}{\rho C_{p}} \frac{\partial^{2} \bar{T}}{\partial \bar{y}^{2}}-\frac{1}{\rho C_{p}} \frac{\partial q_{r}}{\partial y^{\prime}}+\frac{v}{C_{p}}\left(\frac{\partial \bar{u}}{\partial \bar{y}}\right)^{2}
$$

Diffusion equation

$$
\frac{\partial \bar{C}}{\partial \bar{t}}+\bar{v} \frac{\partial \bar{C}}{\partial \bar{y}}=D \frac{\partial^{2} \bar{C}}{\partial \bar{y}^{2}}-\bar{k}\left(\bar{C}-\overline{C_{\infty}}\right)
$$

Where $\bar{T}$ and $\bar{C}$ are the dimensional temperature and concentration, $\rho$ is the fluid density, $v$ is the kinematic viscosity, $C_{p}$ is the specific heat at constant pressure, $\sigma$ is fluid electrical conductivity, $g$ is the acceleration due to gravity, $\beta$ and $\beta^{*}$ are the thermal and concentration expansion coefficients, $B_{0}$ is the magnetic induction, $D$ is the chemical molecular diffusivity, $K_{r}^{\prime}$ is the chemical reaction parameter and $\kappa$ is the fluid thermal conductivity. The first and second terms on the right hand side of the momentum equation (2) denote the thermal and concentration buoyancy effects respectively. 
From (1) asserts that, the suction velocity is either a constant or a function of time. Hence the suction velocity normal to that plate is assumed in the form

$\bar{v}=-v_{0}\left(1+\varepsilon A e^{i \bar{\omega} \bar{t}}\right)$

Where A is real positive constant and $\varepsilon$ is small such as $\varepsilon<<1, \varepsilon A<<1$ and $v_{0}$ is non-zero positive constant. The negative sign indicates that the suction velocity is directed towards the plate.

Using (5) the equations (2), (3) and (4) becomes

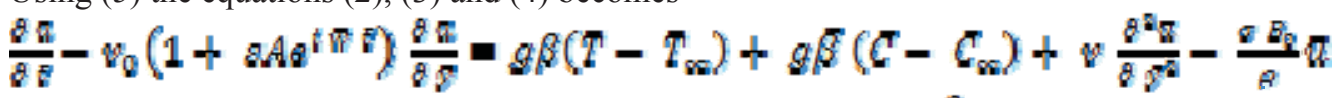

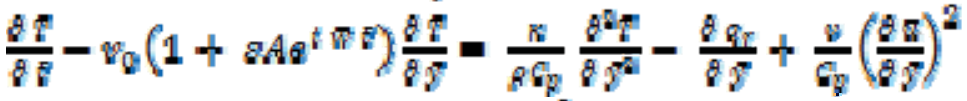

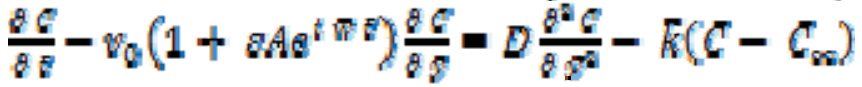

The corresponding initial and boundary conditions of the problems are:

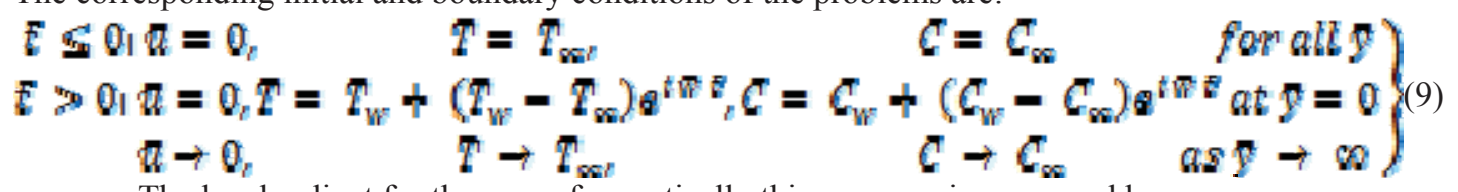

The local radiant for the case of an optically thin gray gas is expressed by

$\frac{8 g_{x}}{8 y}=-4 \alpha^{6} \sigma\left(T_{w}^{4}-T^{4}\right)$

We assume that the temperature difference within the flow are sufficiently small such that $T^{4}$ may be expressed as a linear function of the temperature. This is accomplished by expanding $T^{4}$ is a Taylor series about $T_{\infty}$ and neglecting the higher - order terms, thus

$T^{4} \cong 4 T_{w}^{8} T-3 T_{\mathrm{ou}}^{4}$

By using equations (10) and (11), equation (7) reduces to

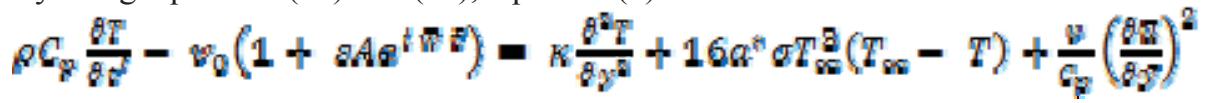

We now introduce the following non-dimensional quantities into the equation (6), (8) and (12)

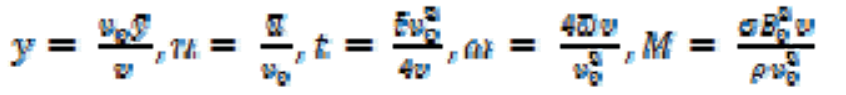

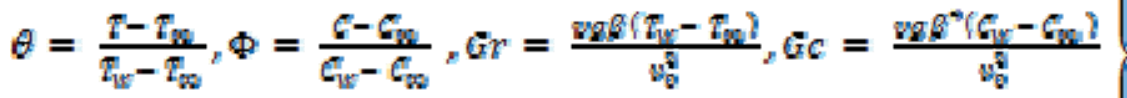

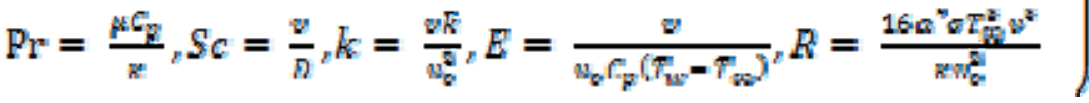

The governing equations (6), (8) and (12) can be rewritten in the non-dimensional form as follows:

$\frac{\partial u}{g v}-\left(1+a A \theta^{i \omega t}\right) \frac{\partial u}{g y}=\frac{g^{2} u}{\partial y^{2}}+G r \theta+G c \Phi-M u$

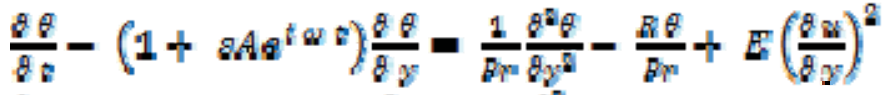

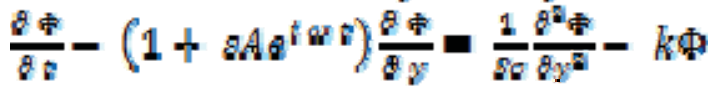

and the initial and boundary conditions are transformed to the following form:

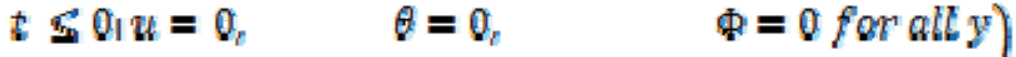

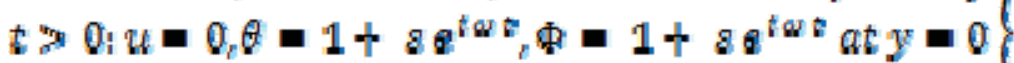

$$
\begin{aligned}
& u \rightarrow 0 \quad \theta \rightarrow 0, \quad \Phi \rightarrow 0 \quad \text { as } y \rightarrow \infty)
\end{aligned}
$$


The mass diffusion equation (16) can be adjusted to represent a destructive reaction (means endothermic i.e. heat is absorbed) if $\mathrm{k}>0$ and generative (means exothermic i.e. heat generated) if $\mathrm{k}<0$.

\section{METHOD OF SOLUTION}

The unsteady, non-linear, coupled partial differential equations (14), (15) and (16) along with their boundary conditions (17) have been solved numerically using an explicit finite difference technique.

The equivalent finite difference scheme of equations for (14), (15) and (16) are as follows:

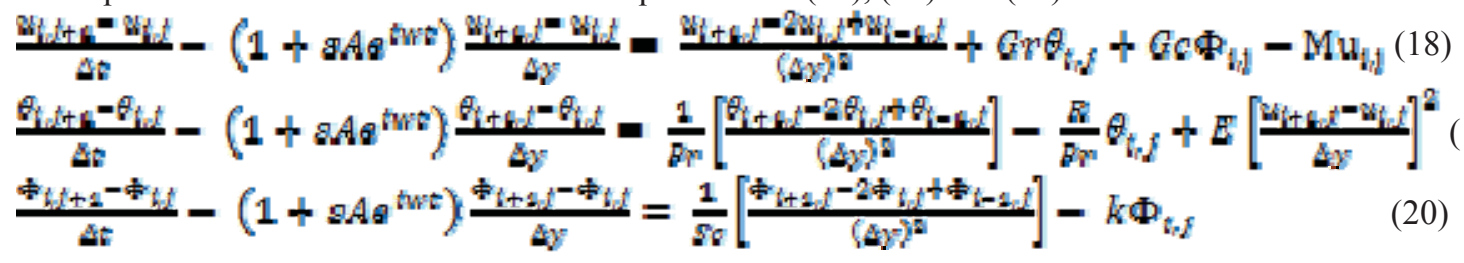

here, index $\mathrm{i}$ refers to $\mathrm{y}$ and $\mathrm{j}$ refers to time, the mesh system is divided by taking $\Delta y=0.1$.

From the initial conditions in (17) we have the following equivalent

$u(t, 0)=0_{v} \theta(t, 0)=0_{z} \Phi(t, 0)=0$ for $a l t$

The boundary conditions from (17) are expressed in finite-difference form as follows

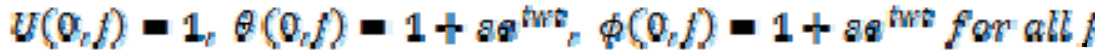

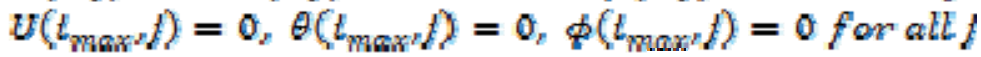

Here infinity is taken as $y=4.1$. First the velocity at the end of the time step namely $y(t, j+1), \mathrm{i}=1$ to 200 is computed from the equation (18) and temperature $\theta(1,+1), \quad \mathrm{i}=1$ to 200 from equation (19) and concentration $\Phi\left(\xi_{n} / \mathbf{1}\right), \mathrm{i}=1$ to 200 from equation $(20)$. The procedure is repeated until $\mathrm{t}=1(\mathrm{i} . \mathrm{e}$., $\mathrm{j}=400)$. During computation $\Delta t$ was chosen as 0.0025 . These computations are carried out for $\operatorname{Pr}=0.71,1.0,7.0,11.4 ; \mathrm{Sc}=$ $0.66,0.94,2.62 ; \mathrm{R}=2.0,4.0,6.0,8.0 ; \mathrm{Gr}=5.0,10.0,15.0,20.0 ; \mathrm{Gc}=5.0,10.0,15.0,20.0 ; \mathrm{E}=0.01,1.00, \mathrm{M}=1,2$, 3. To judge the accuracy of the convergent of the finite difference scheme, the same programmed was run with similar values of $\Delta t$ i.e., $\Delta t=0.0009,0.001$, and no significant change was observed. Hence we conclude that the finite-difference scheme is stable and convergent.

\section{RESULTS AND DISCUSSIONS}

The problem of unsteady free convective flow considering the effect of uniform magnetic field with radiating and chemical reaction is addressed in this study. To have a physical feel of the problem we present results to show how the material parameters of the problem affect the velocity, temperature and concentration profiles. The thermal Grashof number Gr and the Eckert number Ec take positive values corresponds to cooling of the plate by free convection currents. To be realistic, the values of the Schmidt number Sc, so chosen to represent the presence of various species Oxygen $(\mathrm{Sc}=0.66)$, Carbon dioxide $(\mathrm{Sc}=0.94)$ and Propel Benzene at $20^{*} \mathrm{C}(\mathrm{Sc}=2.62)$.
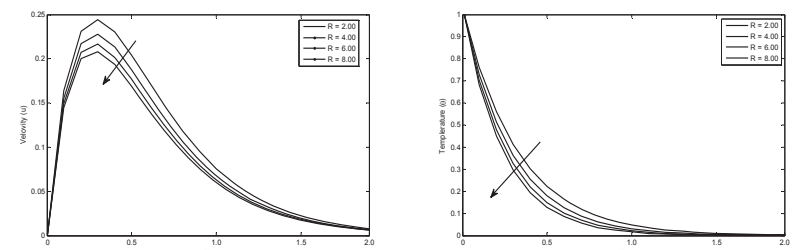

Fig. 1(a \& b) - Velocity and Temperature profiles for different values of Radiation parameter (R)

Figs. 1(a) and 1(b) represents the influence of radiation parameter $\mathrm{R}$ on velocity and temperature. It is noticed that an increase in $\mathrm{R}$ leads to decrease in the velocity and temperature fields. 

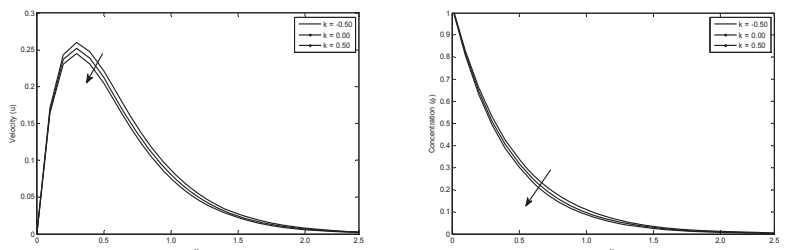

Fig. 2(a \& b) - Velocity and Concentration profiles for different values of Chemical reaction parameter (k)

Figs. 2(a) and 2(b) displays the influence of chemical reaction parameter $\mathrm{k}$ on the velocity and concentration. It is seen, that the effect of increasing values of the chemical reaction parameter $\mathrm{k}$ results in a decreasing velocity distribution as well as concentration also.
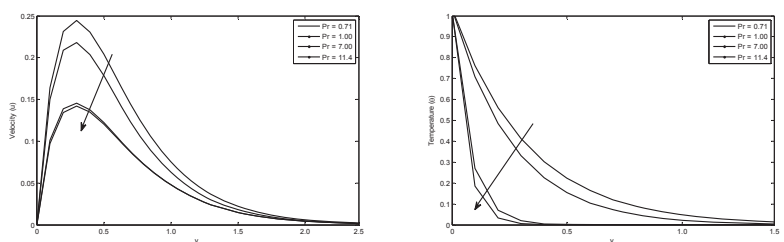

Fig. 3(a \& b) - Velocity and Temperature profiles for different values of Prandtl number ( $\mathrm{Pr}$ )

In Figs. 3(a) and 3(b) we depict the effect of Prandtl number Pr, on the velocity and temperature fields. It is observed that the velocity and temperature both are decreasing with increasing Prandtl number Pr.
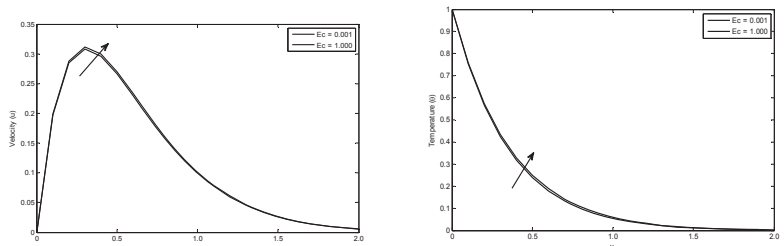

Fig. 4(a \& b) - Velocity and Temperature profiles for different values of Eckert number (Ec)

The influence of Eckert number (Ec) on velocity and temperature profiles are shown in Figs. 4(a) and 4(b). From these figures it is clear that as Eckert number increases velocity and temperature both are increases.
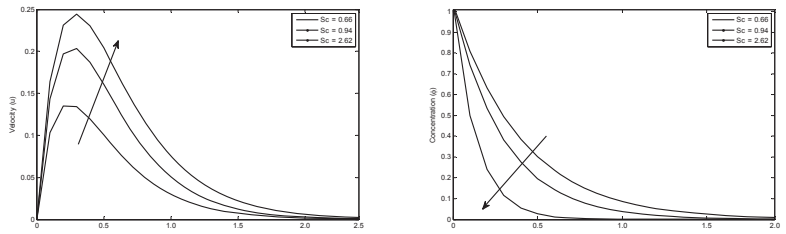

Fig. 5(a \& b) - Velocity and Concentration profiles for different values of Schmidt number (Sc)

Fig. 5(a) and 5(b) illustrate the influence of the Schmidt number Sc on the velocity and concentration fields. It is evident that the velocity and concentration both are decreasing with increasing values of Schmidt number Sc.
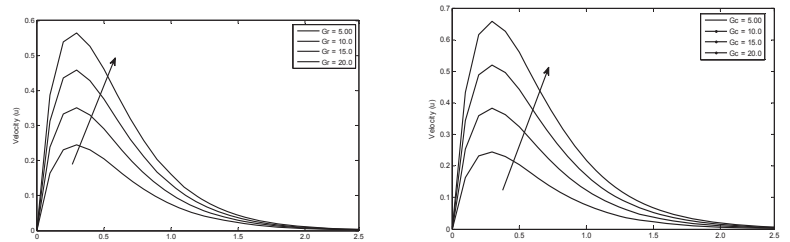

Fig. 6(a \& b) - Velocity profiles for different values of Grashof and modified numbers (Gr and Gc)

The velocity profiles for different values of Grashof number Gr and modified Grashof number Gc are described in Figs. 6(a) and 6(b). It is easily says that velocity increases with increasing values of Grashof number or modified Grashof number. Here the positive values of Gr correspond to a cooling of the surface by natural convection. 


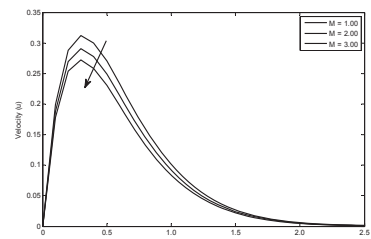

Fig. 7 - Velocity profiles for different values of magnetic parameter (M)

For various values of the magnetic parameter $M$ the velocity profiles are depict in Fig. 7 . The impact of the magnetic parameter $\mathrm{M}$ on the velocity profiles is an expected. velocity decreases with increasing values of $\mathrm{M}$.

\section{CONCLUSIONS}

We summarize below the following results of physical interest on the velocity, temperature and concentration distribution of the flow field.

1. A growing radiation or chemical reaction parameter or magnetic parameter retards the velocity of the flow field at all points.

2. A growing radiation decreases temperature of the flow field at all points

3. The growing chemical reaction parameter decreases the concentration of the flow field at all points.

\section{REFERENCES}

[1] Singh A.K., MHD free convective flow past an accelerated vertical porous plate by finite difference method, Astrophys. Space Sci. 94, pp. 395-400, 1983.

[2] Ahmmed S.F., Das M.K., Ali L.E., Analytical study on MHD free convection and mass transfer flow past a vertical porous plate, American Journal of Applied Mathematics, 3(2), pp.64-74, 2015.

[3] Das S.S., Satapathy A., Das J.K., Panda J.P., Mass transfer effects on MHD flow and heat transfer past a vertical porous plate through porous medium under oscillatory suction and heat source, International Journal of Heat and Mass Transfer, vol. 52, Issues 25-26, December 2009, Pages 5962-5969.

[4] Ahmmed S.F., Mondal S, and Ray A, Numerical studies on MHD free convection and mass transfer flow past a vertical flat plate, IOSR Journal of Engineering, vol. 3, Issue 5, pp. 41-47, May. 2013

[5] Abdus-sattar M.D. and Hamid Kalim M.D., Unsteady free convection interaction with thermal radiation in a boundary layer flow past a vertical porous plate, J. Math. Phys. Sci. 30, pp. 25-37, 1996.

[6] Makinde O.D., Free-convection flow with thermal radiation and mass transfer past a moving vertical porous plate, Int. Comm. Heat Mass Transfer, 32, pp. 1411- 1419, 2005

[7] Abd EL-Naby M.A., El-Barbary E.M.E., and Abdelazem N.Y, Finite difference solution of radiation effects on MHD unsteady freeconvection flow on vertical porous plate, Appl. Math. Comput.151 (2), pp. 327 - 346, 2004.

[8] Mbeledogu I.U., Amakiri A.R.C., and Ogulu A, Unsteady MHD free convection flow of a compressible fluid past a moving vertical plate in the presence of radioactive heat transfer, Int. J. of Heat and Mass Transfer, 50, pp. 1668-1674, 2007.

[9] Muthucumaraswamy R, Chandrakala P., Radiative heat and mass transfer effects on moving isothermal vertical plate in the presence of chemical reaction, Int. J. Appl. Mech. Eng. 11, pp. 639, 2006.

[10] Ibrahim F.S., Elaiw A.M., Bakr A., Effect of the chemical reaction and radiation absorption on the unsteady MHD free convection flow past a semi-infinite vertical permeable moving plate with heat source and suction, Comm. Nonlin. Sci. Numer. Simul. 13, pp. 1056, 2008.

[11] Venkateswarlu M, Padma P, Unsteady MHD free convective heat and mass transfer in a boundary layer flow past a vertical permeable plate with thermal radiation and chemical reaction, Procedia Engineering, 127, pp. $791-799,2015$. 